\title{
Flow pattern transition and destabilization mechanism of thermocapillary convection for low Prandtl number fluid in a deep annular pool with surface heat dissipation
}

\author{
Li Zhang a,b, You-Rong Li ${ }^{\mathrm{a}, *}$, Chun-Mei $\mathrm{Wu}^{\mathrm{a}}$, Qiu-Sheng Liu ${ }^{\mathrm{c}}$

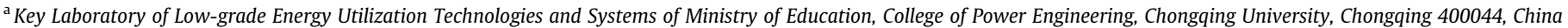 \\ ${ }^{\mathrm{b}}$ Chongqing Nanfang Translators College of Sichuan International Studies University, Chongqing 401120, China \\ ${ }^{\mathrm{c}}$ Key Laboratory of Microgravity (National Microgravity Laboratory), Institute of Mechanics, Chinese Academy of Sciences, Beijing 100190, China
}

\section{A R T I C L E I N F O}

\section{Article history:}

Received 23 November 2017

Received in revised form 28 February 2018

Accepted 24 May 2018

Available online 14 June 2018

\section{Keywords:}

Thermocapillary convection

Low Prandtl number fluid

Surface heat dissipation

Flow pattern transition

Annular pool

\begin{abstract}
A B S T R A C T
In order to understand clearly the flow pattern transition and the destabilization mechanism of thermocapillary convection for low Prandtl number fluids in a deep annular pool with surface heat dissipation, we carried out a series of three-dimensional numerical simulations by using the finite volume method. The radius ratio and the aspect ratio of an annular pool are respectively fixed at 0.5 and 1.0. Prandtl number of the working fluid is 0.011 . Because the total heat dissipation coefficient on the free surface for low Prandtl fluids is small, Biot number is varied from 0 to 1.0. Results indicate that thermocapillary convection experiences the transitions from axisymmetric steady state flow into three-dimensional steady flow, and then into three-dimensional oscillation flow with the increase of Marangoni number. The critical Marangoni number of flow pattern transition decreases slightly with the increase of Biot number, and the maximum temperature and velocity fluctuations appear near the lower part of outer wall. The azimuthal temperature fluctuation on the free surface gradually shrinks to the inner wall, and the temperature fluctuation region decreases. However, it remains almost unchanged near the bottom of the annular pool, but the fluctuation amplitude increases.
\end{abstract}

(c) 2018 Elsevier Ltd. All rights reserved.

\section{Introduction}

Thermocapillary convection driven by surface tension gradient has received considerable attention for its rich dynamical behaviors and complex flow pattern transition processes. For thermocapillary convection with an adiabatic free surface, many fruitful research results have been achieved [1-5]. As early as 1983, Smith and Davis [6,7] discussed the destabilization mechanisms by using linear stability theory in an infinite liquid layer and showed that stationary longitudinal rolls and unsteady hydrothermal waves (HTWs) for the case with flat and non-deformable free surface, and surface waves for the case with deformable free surface. Furthermore, the formation mechanism of hydrothermal waves is explained by Smith [8]. Subsequently, Zebib et al. [9] and Ben Hadid and Roux [10] presented numerical results concerning thermocapillary flows in cavities with different aspect ratios, while Mercier and Normand [11] and Peng et al. [12] performed numerical simulations for rectangular and annular pools, respectively.

\footnotetext{
* Corresponding author.

E-mail address: liyourong@cqu.edu.cn (Y.-R. Li).
}

Villers and Platten [13] investigated experimentally and numerically buoyancy-thermocapillary convection in acetone solution and found that the flow will experience the processes from single vortex steady flow into multi vortex steady flow, and into unsteady flow with the increase of Marangoni number. Zhu et al. [14] observed various dynamic states in a rectangular pool with the applied temperature difference between the two sidewalls adjusted in the range of $(0-43){ }^{\circ} \mathrm{C}$ by the experiment, and discussed the relationship between oscillatory frequency and Marangoni number. Gillon and Homsy [15] used particle image velocimetry (PIV) to analyze buoyancy-thermocapillary convection of $0.65 \mathrm{cSt}$ silicone oil layer in a rectangular pool and explained the influence of Marangoni effect on flow pattern transition. Chen et al. [16] and Yu et al. [17] carried out the numerical simulation to analyze the physical mechanism of the hydrothermal wave formation for the low $(P r=0.011)$ and moderate $(P r=6.7)$ Prandtl number fluids in an annular liquid pool. What's more, some scholars have experimentally observed the stationary longitudinal roll pattern and the HTWs in an annular pool [18-22].

Owing to the interfacial non-equilibrium effect, heat dissipation to the environment on the free surface becomes inevitable. 


\begin{tabular}{|c|c|c|}
\hline \multicolumn{3}{|c|}{ Nomenclature } \\
\hline$B i$ & Biot number & Greek symbols \\
\hline$d$ & depth of annular pool, $\mathrm{m}$ & $\alpha \quad$ thermal diffusivity, $\mathrm{m}^{2} / \mathrm{s}$ \\
\hline$F$ & dimensionless frequency & aspect ratio \\
\hline$h$ & convective heat transfer coefficient, $\mathrm{W} /\left(\mathrm{m}^{2} \cdot \mathrm{K}\right)$ & temperature coefficient of surface tension, $\mathrm{N} /(\mathrm{m} \cdot \mathrm{K})$ \\
\hline$m$ & wave number & thermal conductivity, $\mathrm{W} /(\mathrm{m} \cdot \mathrm{K})$ \\
\hline$M a$ & Marangoni number & radius ratio \\
\hline$p$ & pressure, $\mathrm{Pa}$ & dynamic viscosity, $\mathrm{kg} /(\mathrm{m} \cdot \mathrm{s})$ \\
\hline$P$ & dimensionless pressure & kinematic viscosity, $\mathrm{m}^{2} / \mathrm{s}$ \\
\hline $\operatorname{Pr}$ & Prandtl number & dimensionless temperature \\
\hline$r$ & radius, $\mathrm{m}$ & density, $\mathrm{kg} / \mathrm{m}^{3}$ \\
\hline$R$ & dimensionless radius & dimensionless time \\
\hline$t$ & time, s & dimensionless stream function \\
\hline$T$ & temperature, $\mathrm{K}$ & \\
\hline$u$ & radial velocity, $\mathrm{m} / \mathrm{s}$ & Subscripts \\
\hline$U$ & dimensionless radial velocity & $0 \quad$ ambient \\
\hline$v$ & azimuthal velocity, m/s & inner \\
\hline V & dimensionless azimuthal velocity & outer \\
\hline $\boldsymbol{V}$ & dimensionless velocity vector & period \\
\hline$w$ & axial velocity, $\mathrm{m} / \mathrm{s}$ & \\
\hline W & dimensionless axial velocity & \\
\hline$z$ & axial coordinate, $\mathrm{m}$ & \\
\hline$Z$ & dimensionless axial coordinate & \\
\hline
\end{tabular}

Therefore, the effect of heat dissipation on thermocapillary convection should be considered. Kuhlmann and Albensoeder [23] presented a linear stability analysis on the buoyant-thermocapillary flow in an open rectangular cavity with various aspect ratios. A stationary three-dimensional (3D) cellular flow was obtained at a small aspect ratio, while for a large aspect ratio, hydrothermal waves appeared. Peltier and Biringen [24] provided a stability diagram as a function of aspect ratio for the fluid with $\operatorname{Pr}=6.78$ in two-dimensional (2D) rectangular cavities. They found that a critical aspect ratio is near 2.3 and the minimum critical Marangoni number is near 20,000. Sab et al. [25] investigated 3D steady thermocapillary convection in an open cubic container and showed the damping effect of the front and back walls on the temperature distribution by comparing 3D with 2D simulation results in steady state flows. Jing et al. [26] analyzed the mechanism of the wellknown surface spoke patterns in an open crucible. It was certified that the spoke patterns on the free surface are caused by Marangoni instability. Sim and Zebib [27] reported a series of 3D numerical simulation results on thermocapillary convection in an open cylindrical annulus. They found that there were two disjoint neutral curves when $\operatorname{Pr} \leq 4.4$, and heat loss from the free surface provided an explanation for the dependence of the critical Marangoni number on the container size at a fixed aspect ratio which was observed in the experiments by Kamotani et al. [28]. Schwabe et al. [29] reported the results of microgravity experiments on thermocapillary convection in open annuli with outer radius of $40 \mathrm{~mm}$, inner radius of $20 \mathrm{~mm}$ and various depth. They discussed the supercritical oscillation periods and the oscillations of hydrothermal waves at the large aspect ratio. It was found that the hydrothermal waves exhibit an internal corotating multicellular pattern. Sim et al. [30] investigated numerically oscillatory thermocapillary convection in open cylindrical annuli heated from the outer wall. Results show that the transition to oscillatory states occurs at critical thermocapillary Reynolds number which depends on the aspect ratio. Heat loss from the free surface or heat input from the surroundings to the free surface stabilizes the flow, and the critical thermocapillary Reynolds number increases with the increase of Biot number while the critical period goes down. The numerical results agree better with the experimental ones if the free surface is assumed to be heated. Oztop et al. [31] reported the numerical results on the coupled buoyancy and thermocapillary convection and performed the evaluation of entropy generation. They found that Marangoni number becomes more effective parameter on total entropy generation at lower Rayleigh numbers. Sakhy et al. [32] presented the numerical work on Rayleigh-Béna rd-Marangoni convection in an open cylindrical container heated by a non-uniform flux. It was found that the flow pattern depends on Rayleigh number, Biot number, Marangoni number and the ratio of the thermal conductivities of the solid substrate and the fluid. Hoyas et al. [33-35] studied the instabilities in a cylindrical annulus with the heating bottom and the opening free surface to the atmosphere by linear stability analysis. It was found that Marangoni number, Biot number and Prandtl number become the key factors affecting the various flow patterns after the flow destabilization and the flow bifurcation routes to chaos.

In our previous works [36,37], we reported a series of numerical simulation results on the effect of surface heat dissipation on thermocapillary convection in a shallow annular pool. In this case, with the increase of heat dissipation, the flow has gone through two bifurcations and transits to different flow patterns, including hydrothermal waves, radial moving waves and coexisting hydrothermal waves and radial moving waves, and so on. This paper presented another sets of numerical simulations to explore the flow pattern transition and destabilization mechanism of thermocapillary convection for low Prandtl number fluids in a deep annular pool with surface heat dissipation, which is very different from those in a shallow annular pool.

\section{Problem statement}

\subsection{Basic assumptions and governing equations}

Fig. 1 provides the physical model and the coordinate system for the problem. A deep annular pool with inner radius $r_{\mathrm{i}}$, outer radius $r_{\mathrm{o}}$ and depth $d$ is filled with the low Prandtl number fluid $(P r=0.011)$. The radius ratio and the aspect ratio of the annular pool are respectively defined as $\eta=r_{\mathrm{i}} / r_{\mathrm{o}}$ and $\varepsilon=d /\left(r_{\mathrm{o}}-r_{\mathrm{i}}\right)$. 


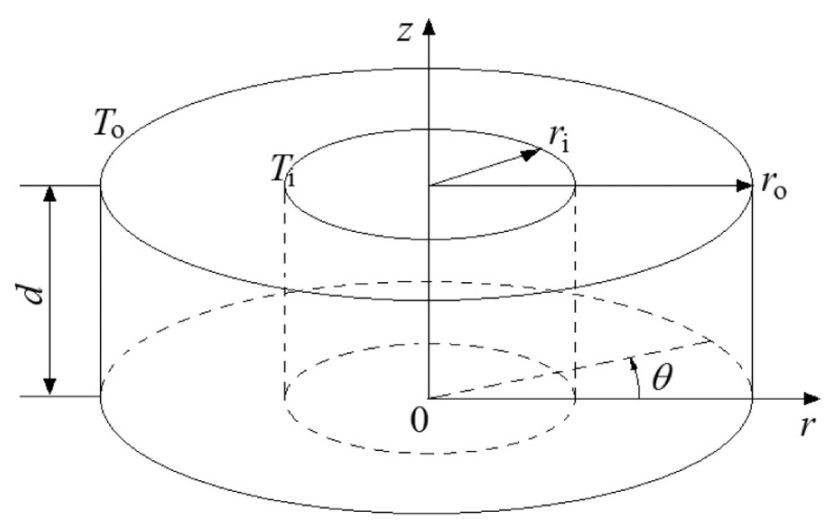

Fig. 1. Physical model and the coordinate system.

The bottom is adiabatic, which satisfies no-slip and impermeable boundary conditions. At the top free surface, heat dissipation to ambient was taken into account. The horizontal free surface is assumed to be flat and non-deformable. The total surface heat transfer coefficient is marked by $h$. The inner and outer cylinders are isothermally maintained at specific temperatures $T_{\mathrm{i}}$ and $T_{\mathrm{o}}$ $\left(T_{\mathrm{o}}>T_{\mathrm{i}}\right)$, respectively.

In order to simplify the problem, the following basic assumptions is introduced. (1) The fluid is an incompressible Newtonian fluid. The thermophysical properties are all constant except for the surface tension. (2) The velocity is small, and the flow is laminar. (3) The free surface is flat and non-deformable. (4) If there is evaporation cooling on the free surface, the evaporation rate is too slow to affect the depth of the liquid layer.

By applying $\left(r_{\mathrm{o}}-r_{\mathrm{i}}\right), v /\left(r_{\mathrm{o}}-r_{\mathrm{i}}\right),\left(r_{\mathrm{o}}-r_{\mathrm{i}}\right)^{2} / v$ and $\mu v /\left(r_{\mathrm{o}}-r_{\mathrm{i}}\right)^{2}$ as scale quantities for length, velocity, time and pressure, respectively, we have

$$
\begin{aligned}
& (R, Z)=\frac{(r, z)}{r_{o}-r_{i}},(U, V, W)=\frac{(u, v, w)}{v /\left(r_{o}-r_{i}\right)}, \tau=\frac{t}{\left(r_{o}-r_{i}\right)^{2} / v}, \\
& P=\frac{p}{\mu \nu /\left(r_{o}-r_{i}\right)^{2}}
\end{aligned}
$$

Based on the above assumptions, the governing equations of continuity, momentum and energy together with the proper boundary conditions can be expressed by the following forms:

$\nabla \cdot \boldsymbol{V}=0$

$\frac{\partial \boldsymbol{V}}{\partial \tau}+\boldsymbol{V} \cdot \nabla \boldsymbol{V}=-\nabla P+\nabla^{2} \boldsymbol{V}$

$\frac{\partial \Theta}{\partial \tau}+\boldsymbol{V} \cdot \nabla \Theta=\frac{1}{P r} \nabla^{2} \Theta$

In the formula, $\boldsymbol{V}, \tau$ and $P$ respectively represented the dimensionless velocity vector, time and pressure. $\Theta$ is the dimensionless temperature, which is defined as

$\Theta=\left(T-T_{i}\right) /\left(T_{o}-T_{i}\right)$

Here shows the initial conditions:

$\tau=0, U=V=W=0, \Theta=-\ln [R(1-\eta) / \eta] / \ln \eta$

According to the above assumptions, the following boundary conditions are considered:

At the inner cylinder $\left(R=R_{\mathrm{i}}=r_{\mathrm{i}} /\left(r_{\mathrm{o}}-r_{\mathrm{i}}\right)=\eta /(1-\eta), 0 \leq Z \leq \varepsilon\right)$ : $U=V=W=0, \Theta=0$;

At the outer cylinder $\left(R=R_{\mathrm{o}}=r_{\mathrm{o}} /\left(r_{\mathrm{o}}-r_{\mathrm{i}}\right)=1 /(1-\eta), 0 \leq Z \leq \varepsilon\right)$ : $U=V=W=0, \Theta=1$;
At the bottom $(Z=0, \eta /(1-\eta)<R<1 /(1-\eta))$ :

$U=V=W=0, \partial \Theta / \partial Z=0$.

At the free surface $(Z=\varepsilon, \eta /(1-\eta)<R<1 /(1-\eta))$, the radial velocity $U$ and the azimuthal velocity $V$ depend on the balance effect between the thermocapillary force and the shear stress of the fluid. At the same time, the axial velocity caused by surface evaporation is too small to be neglected. Therefore,

$\frac{\partial U}{\partial Z}=-\frac{M a}{\operatorname{Pr}} \frac{\partial \Theta}{\partial R}, \frac{\partial V}{\partial Z}=-\frac{M a}{\operatorname{Pr}} \frac{\partial \Theta}{R \partial \theta}, W=0$,

where $M a$ is Marangoni number, $M a=\gamma_{\mathrm{T}} \Delta T\left(r_{\mathrm{o}}-r_{\mathrm{i}}\right) /(\mu \alpha), \gamma_{\mathrm{T}}$ surface tension temperature coefficient, $\mu$ dynamic viscosity, $\alpha$ thermal diffusivity.

The thermal boundary condition on the free surface can be expressed as

$-\lambda \partial T / \partial z=h\left(T-T_{0}\right)$

where $\lambda$ is thermal conductivity. Supposing $T_{0}=T_{\mathrm{i}}$, Eq. (10) is also expressed in the dimensionless form

$-\partial \Theta / \partial Z=B i \Theta$.

where $B i$ is surface heat dissipation Biot number, $B i=h\left(r_{\mathrm{o}}-r_{\mathrm{i}}\right) / \lambda$.

\subsection{Calculation conditions and numerical method}

In this work, the radius ratio and the aspect ratio of the annular pool are respectively fixed at $\eta=0.5$ and $\varepsilon=1.0$. Surface heat dissipation Biot number is within the range of $B i=0-1.0$. Marangoni number is gradually increased from $10^{2}$ to $10^{3}$.

For the discretization of the fundamental equations, the finite volume method is adopted. The central-difference scheme is for the diffusion terms while the convective terms are treated by the QUICK scheme. The SIMPLE algorithm is used for correcting simultaneously the pressure and the velocity. The dimensionless time step varies in the range between $0.5 \times 10^{-3}$ and $2 \times 10^{-3}$. During the whole iterative process for solving, if the maximum relative error of temperature and velocity is less than $10^{-5}$, we considered that the iterative method has to be convergent to a solution.

Non-uniform staggered grid of $52^{\mathrm{R}} \times 52^{\mathrm{Z}} \times 180^{\theta}$ with denser meshes near the solid walls and the free surface than those in other regions is applied. To verify the convergence of the mesh, the numerical simulation is carried out at four different grids of $32^{\mathrm{R}}$ $\times 32^{\mathrm{Z}} \times 90^{\theta}, 42^{\mathrm{R}} \times 42^{\mathrm{Z}} \times 120^{\theta}, 52^{\mathrm{R}} \times 52^{\mathrm{Z}} \times 180^{\theta}$ and $62^{\mathrm{R}} \times 62^{\mathrm{Z}} \times$ $240^{\theta}$ at $M a=400$ when $B i=0.0,0.5$ and 1.0 , respectively. Results indicate that the flow patterns in all four grids are the same. The relative deviations of azimuthal wave number $m$ and the maximum azimuthal dimensionless temperature fluctuation between grids $52^{\mathrm{R}} \times 52^{\mathrm{Z}} \times 180^{\theta}$ and $62^{\mathrm{R}} \times 62^{\mathrm{Z}} \times 240^{\theta}$ are all less than $5 \%$. When both the numerical accuracy and the computational expense are taken into account, the grid of $52^{\mathrm{R}} \times 52^{\mathrm{Z}} \times 180^{\theta}$ is chosen for all cases. On the other hand, the code version for 3D numerical simulation of thermocapillary convection has been verified in the previous papers [12,16-18,36-38].

\section{Results and discussion}

When the temperature gradient exists on the free surface, the surface tension gradient will drive the fluid flowing from the high temperature wall to the low temperature wall along the free surface. If the temperature difference between inner wall and outer wall is small, thermocapillary convection is two-dimensional, steady and axisymmetric. In this case, the flow field can be expressed by the streamlines in the $R-Z$ plane. The dimensionless flow function is defined as 
$U=\frac{1}{R} \frac{\partial \psi}{\partial Z}, W=-\frac{1}{R} \frac{\partial \psi}{\partial R}$

Fig. 2 reveals the distributions of isotherms and streamlines under different Biot numbers at $M a=180$. It can be found that the whole annular pool is occupied by a large thermocapillary convection cell, and the center of the flow cell is almost located in the center of the liquid pool. However, for high Prandtl fluids, the center of the flow cell in the annular pool is closer to the free surface and the flow near the bottom of the liquid pool is very weak, as shown in Refs. $[30,38]$. Therefore, Prandtl number of the working fluid has an important influence on the flow cell structure. When Biot number is small, the radial temperature gradient on the free surface near the inner wall is larger, the flow is stronger, and the streamlines are denser. With the increase of Biot number, the free surface temperature near the inner wall drops, the radial temperature gradient decreases gradually and the thermocapillary effect weakens. However, near the outer wall, the radial temperature gradient and the thermocapillary force increase, and thus the thermocapillary convection cell moves outward slightly, which is different from the results in a shallow annular pool [36,37]. Meanwhile, the flow intensity strengthens continuously, and the dimensionless flow function increases.

With the increase of Marangoni number, the flow strengthens continuously. When Marangoni number exceeds some critical value, two-dimensional axisymmetric flow will first be transformed into 3D steady flow, and then into 3D oscillatory flow with the further increase of Marangoni number no matter Biot number. However, in a shallow annular pool, two-dimensional axisymmetric flow will directly bifurcate to 3D oscillatory flow at a strong heat dissipation $[36,37]$. Because the maximum temperature gradient appears near the inner wall on the free surface, the flow begins to lose its stability near the inner wall. Fig. 3 gives the
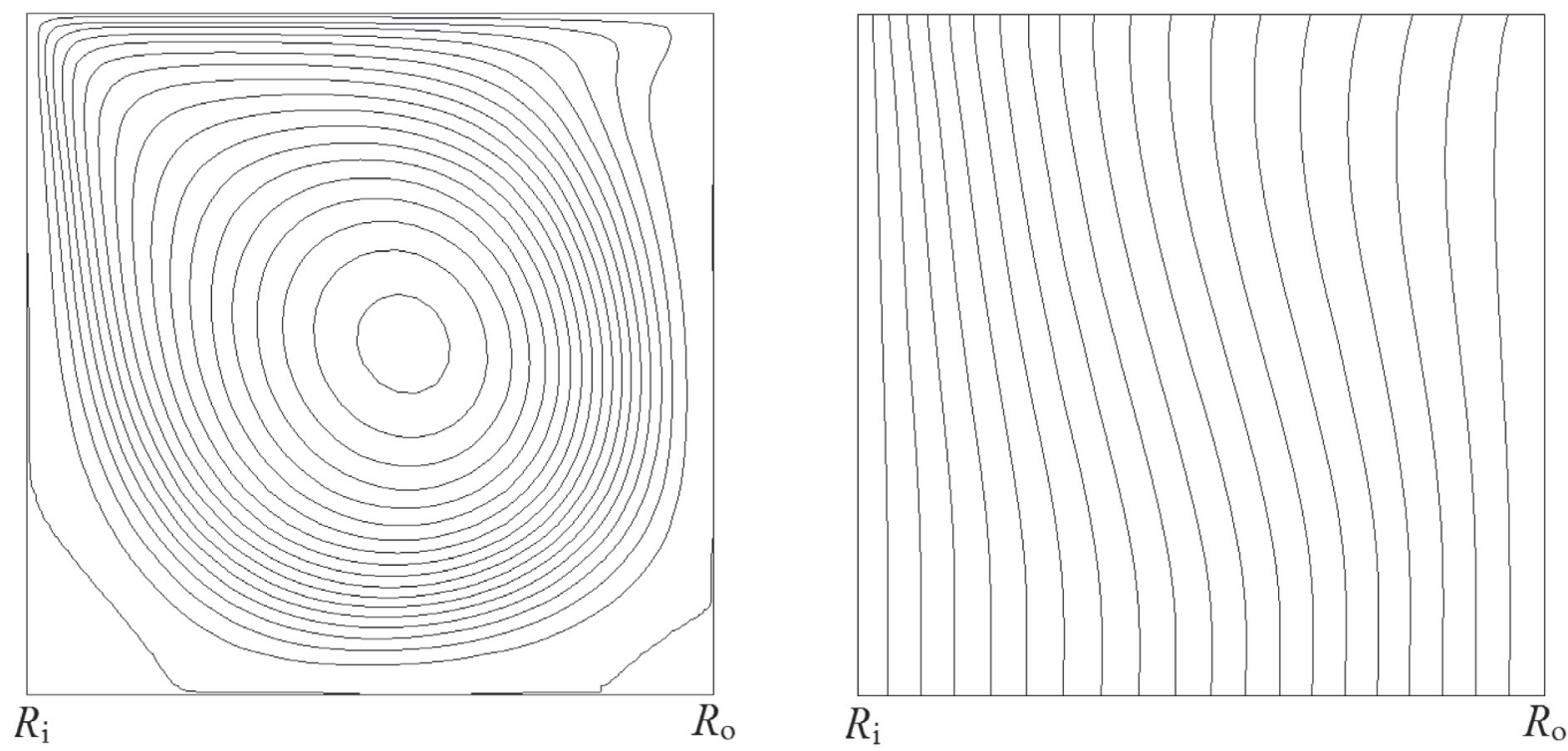

(a) $B i=0.5, \psi_{\max }=92$
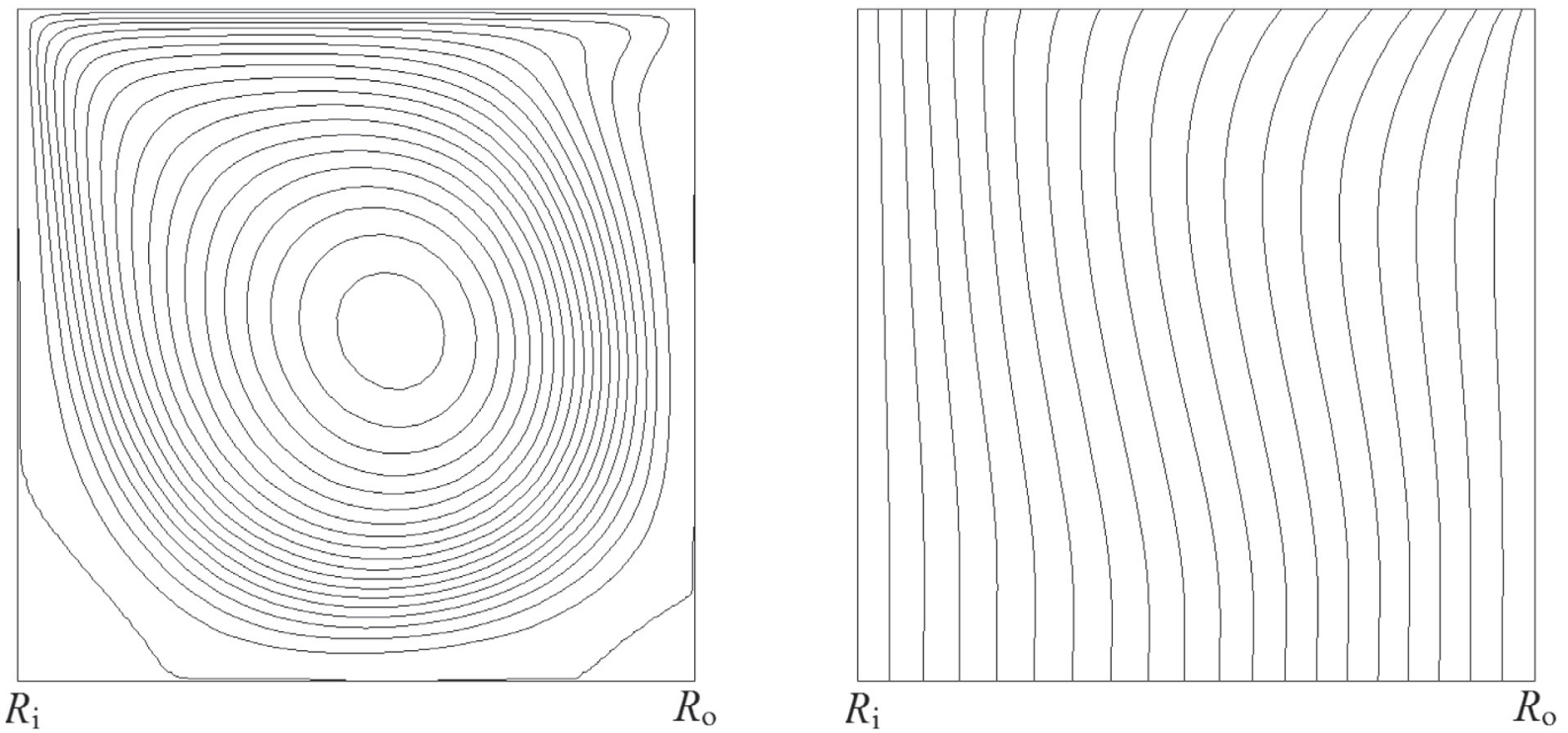

(b) $B i=1.0, \psi_{\max }=98$

Fig. 2. Streamlines (left) and isotherms (right) of $2 \mathrm{D}$ axisymmetric steady flow in a meridional plane at $M a=180 . \delta \Theta=0.05, \delta \psi=5$. 


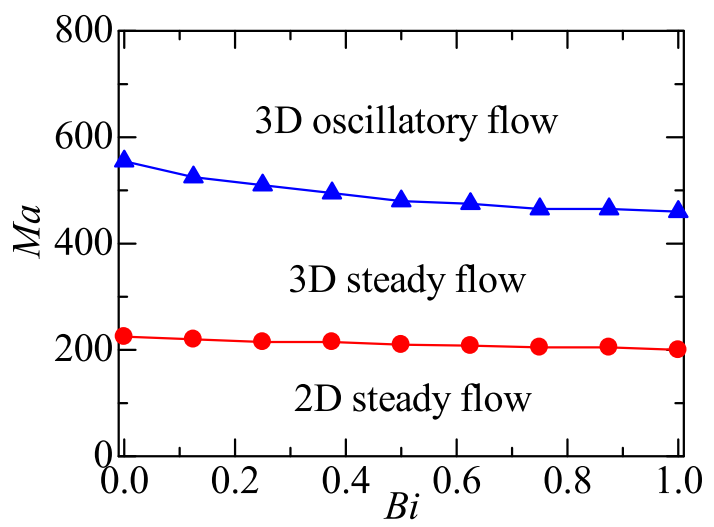

Fig. 3. Variation of the critical Marangoni numbers of the flow pattern transition with Biot number.

critical Marangoni numbers of two flow pattern transition. Obviously, with the increase of surface heat dissipation Biot number, the average radial temperature gradient on the free surface increases and the flow strengthens, so the flow is more prone to instability, and the corresponding critical Marangoni number also decreases gradually.

In order to analyze the distribution of temperature fluctuation in $3 \mathrm{D}$ steady flow, the azimuthal temperature fluctuation $\delta \Theta$ is introduced, which is defined as

$\delta \Theta=\Theta(R, Z, \theta, \tau)-\frac{1}{2 \pi} \int_{0}^{2 \pi} \Theta(R, Z, \theta, \tau) \mathrm{d} \theta$

Fig. 4 shows the dimensionless temperature fluctuation distribution at different cross sections in 3D steady flow. When the fluid flowing along the free surface touches the inner wall, the flow direction becomes downward, as shown in Fig. 2. The sudden change of flow direction will arouse the temperature fluctuation along the azimuthal direction. Therefore, the temperature fluctua- tion on the free surface mainly appears near the inner wall, and the temperature distribution along the azimuthal direction near the outer wall is almost uniform. On the contrary, near the bottom of the liquid pool, after the outward radial flow meets the outer wall, the flow direction also changes suddenly, so that the azimuthal temperature fluctuation near the bottom of the liquid pool occurs mainly near the outer wall, and the temperature distribution along the azimuthal direction near the inner wall is almost even. Comparing the temperature fluctuations at the bottom and on the free surface of the liquid pool, it can be found that wave numbers along the azimuthal direction are the same and azimuthal positions are also synchronous, although radial positions of temperature fluctuations are different. In the middle of liquid pool, that is, the cross section of $Z=0.5$, a new temperature fluctuation pattern is induced because of the shear action between the downward and upward flows, which has the same wave number with that at the bottom and on the surface, but a staggered azimuthal position with half a wave, as shown in the dashed line region in Fig. 4. Comparison of Fig. 4 (a) and (b), it can be seen that with the increase of Biot number, the azimuthal temperature fluctuation on the free surface gradually shrinks to the inner wall, the temperature fluctuation region decreases. However, the temperature fluctuation region remains unchanged at the bottom, and the fluctuation amplitude increases. On the other hand, Biot number has slight effect on wave number. With the same Marangoni number, the variation of wave number is no more than 2 when Biot number increases from 0 to 1.0 .

Fig. 5 shows the variation of the maximum temperature fluctuation $\delta \Theta_{\max }$ in transverse cross section with axial position $Z$ at different Biot numbers. The azimuthal temperature fluctuation mainly originates from the sudden change of flow direction near the top inner wall and the bottom outer wall, where the strong shear of the downward and upward flows are generated. As a result, the temperature fluctuates greatly near the bottom and the top, while it fluctuates slightly in the middle because of the weak shear. On the other hand, the flow satisfies the non-slip condition at the bottom of the liquid pool, but the top is free surface, so
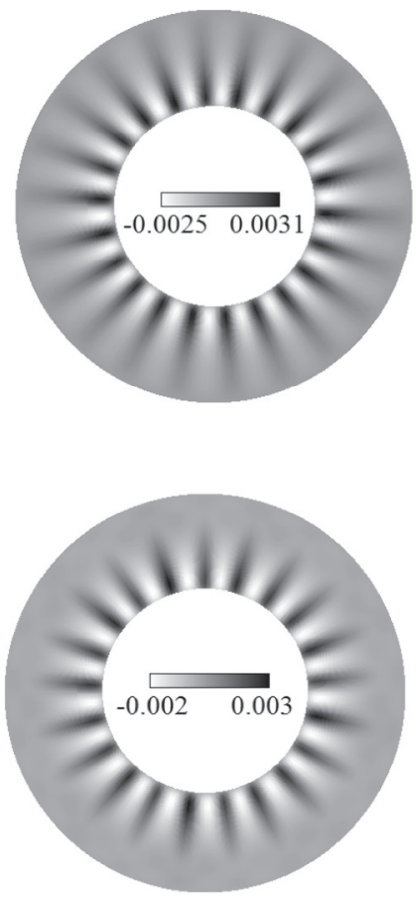

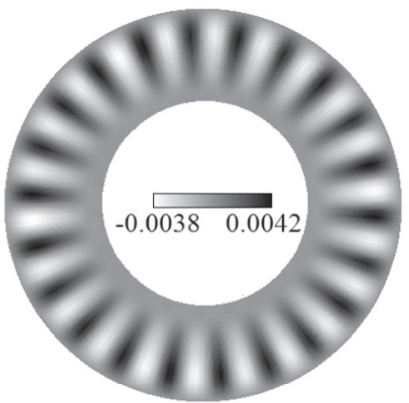

(a) $B i=0$
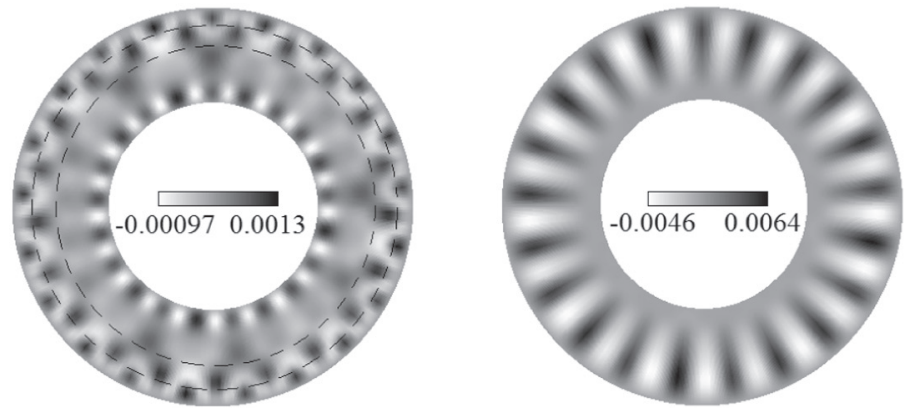

(b) $B i=1.0$

Fig. 4. Azimuthal temperature fluctuation of 3D steady flow on $Z=1.0$ (left), 0.5 (middle) and 0.0 (right) at $M a=400$. 


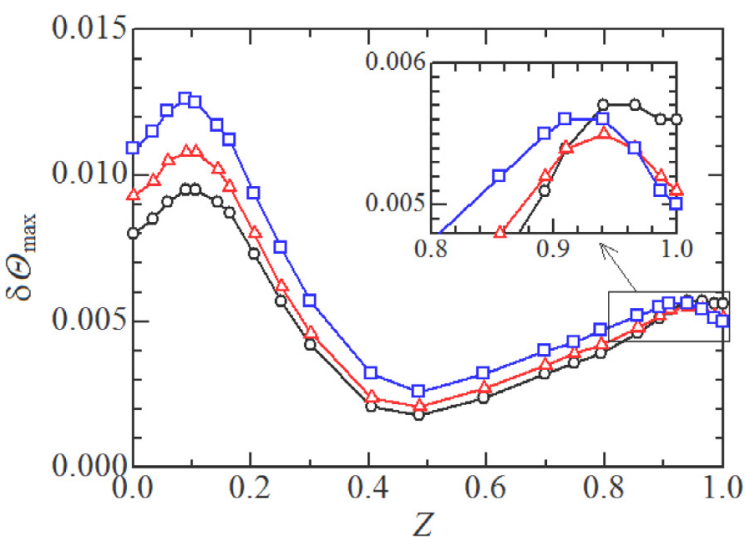

Fig. 5. Variation of the maximum temperature fluctuation in transverse cross section with the axial position. Circle: $B i=0.0$; triangle: $B i=0.5$; square: $B i=1.0$.

velocity gradient is large near the bottom of the liquid pool and small near the free surface, so that the temperature fluctuation near the bottom is about twice as large as that near the free surface. At the same time, with the increase of Biot number, the flow in the liquid pool will be enhanced, so the temperature fluctuation amplitude will totally increase. However, the increase of Biot num- ber will result in the decrease of the radial temperature gradient near the inner wall on the free surface, so the temperature fluctuation amplitude on the free surface will decrease slightly, as shown in partial enlarged detail in Fig. 5.

Fig. 6 reveals the velocity vector field and the isotherms of 3D steady flow on $Z-\theta$ section of $0<\theta<\pi / 4$ at $R=1.25$ and 1.75 when $M a=400$ and $B i=0.5$. Obviously, near the inner wall, except for the downward main flow, only in the vicinity of the bottom there is the secondary flow in the azimuthal direction, and away from the bottom the secondary flow is weak. The convection toward the bottom has little effect on the azimuthal temperature distribution, so the azimuthal temperature fluctuation at $R=1.25$ is mainly located near the free surface. Near the outer wall, except for the upward main flow, the secondary flow region near the bottom expands upward, and the flow is enhanced. Therefore, the azimuthal temperature fluctuation near the bottom at $R=1.75$ increases. Because the bottom is the adiabatic boundary, the azimuthal temperature distribution depends mainly on the velocity distribution of outward backflow near the bottom. Therefore, although the directions of the secondary flows on the free surface and near the bottom are opposite, the temperature fluctuation is synchronous in the azimuthal direction.

When Marangoni number exceeds the second critical value, 3D steady flow will destabilize and transform into 3D oscillatory flow. In order to analyze the variation of temperature fluctuation in $3 \mathrm{D}$
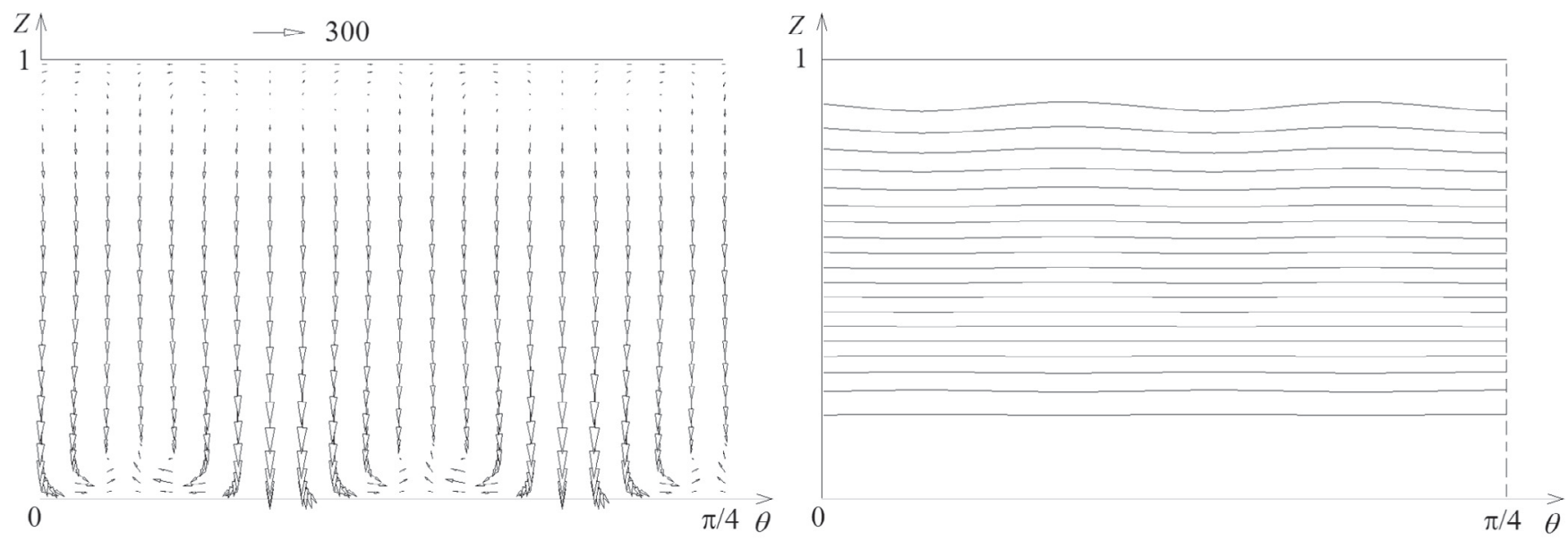

(a) $R=1.25, \Theta_{\max }=0.2217, \Theta_{\min }=0.4147, \delta \Theta=\left(\Theta_{\max }-\Theta_{\min }\right) / 20$
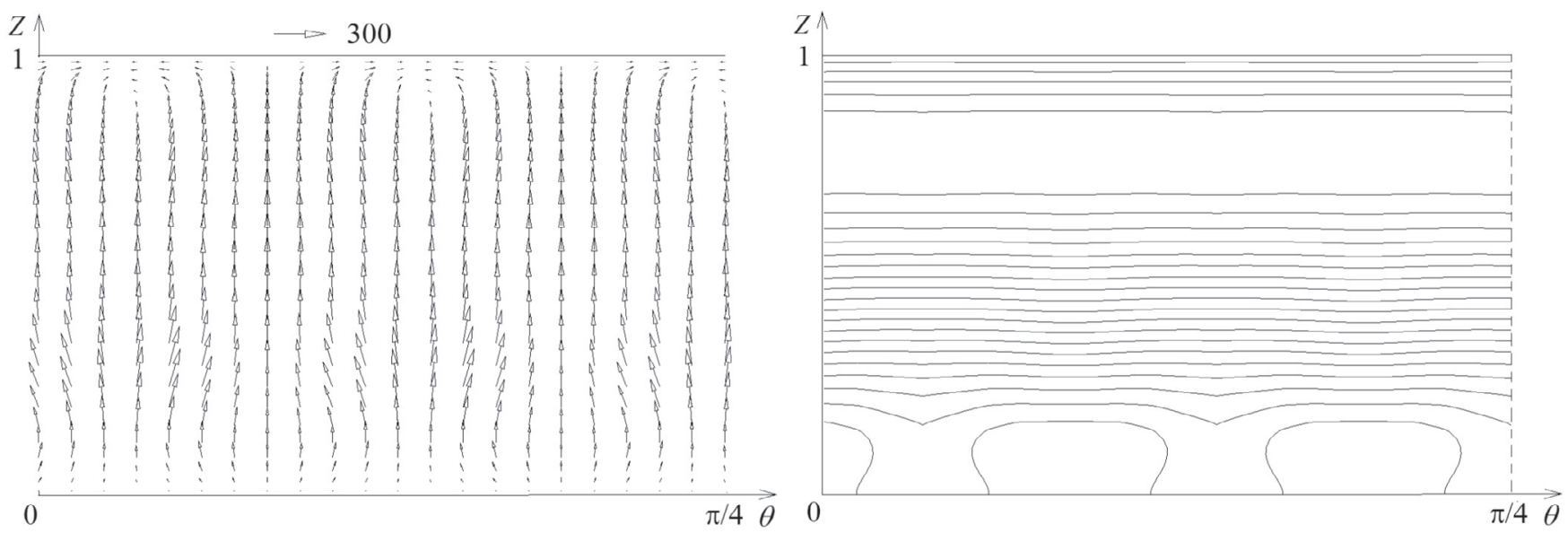

(b) $R=1.75, \Theta_{\max }=0.8166, \Theta_{\min }=0.6929, \delta \Theta=\left(\Theta_{\max }-\Theta_{\min }\right) / 20$

Fig. 6. Velocity vector field (left) and isotherms (right) of 3D steady flow on $Z-\theta$ section of $0<\theta<\pi / 4$ at $M a=400$ and $B i=0.5$. 
oscillatory flow, the time fluctuation of temperature $\delta \Theta$ is introduced as

$\delta \Theta=\Theta(R, \theta, Z, \tau)-\frac{1}{\tau_{\mathrm{p}}} \int_{0}^{\tau_{\mathrm{p}}} \Theta(R, \theta, Z, \tau) \mathrm{d} \tau$

in which, $\tau_{\mathrm{p}}$ is the fluctuation period.

Fig. 7 shows the snapshots of temperature fluctuation in the $R-Z$ plane of $\theta=0$ within a period for 3D oscillatory flow at $M a=500$ and $B i=0.5$. It is shown from the diagram that the temperature fluctuations originate near the bottom, and then propagate to the free surface. During the propagating process, the temperature fluctuation amplitude decreases first and then increases. It is further proved that the flow instability is mainly caused by the sudden change of flow direction near the upper inner wall and the bottom outer wall in a deep pool for low Prandtl number fluids. In a shallow pool, the flow destabilization should be attributed to the HTWs instability $[6,7,36,37]$. Therefore, the aspect ratio of the pool is an important factor for the flow destabilization mechanism. On the other hand, Jing et al. [26] investigated thermocapillary convection of LiNbO3 melt with Prandtl number of 13.6 in an open cylindrical liquid pool with surface radiation heat dissipation. The results show that Marangoni effect on the free surface is the main cause of the surface wheel pattern formation, and the fluctuations of temperature and velocity only occur near the free surface. At the same time, Li et al. [38] numerically simulated thermocapillary convection of $0.65 \mathrm{cSt}$ silicone oil $(\mathrm{Pr}=6.7)$ in the annular pool and showed that the fluctuations of temperature and velocity originate from the boundary layer near the wall. Therefore, Prandtl number of the working fluid should be responsible for the destabilization mechanism of thermocapillary convection in a deep annular pool.

Fig. 8 gives the snapshots of temperature fluctuation within a period for 3D oscillatory flow on the free surface and at the bottom when $M a=500$ and $B i=0.5$. Obviously, the temperature fluctuation includes the azimuthal temperature fluctuation and the radial temperature fluctuation of time-dependent caused by large cells in the $R-Z$ plane. On the free surface, the azimuthal temperature fluctuation exists near the inner wall, and the azimuthal position is stable. But near the outer wall, the time-dependent radial

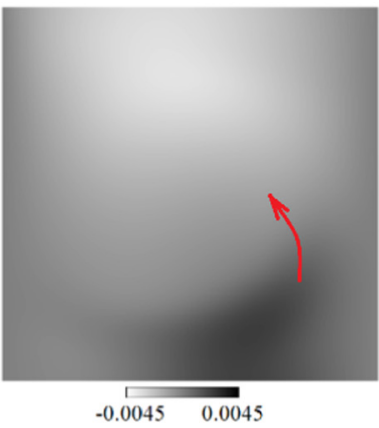

(a) $\tau=\tau_{0}$

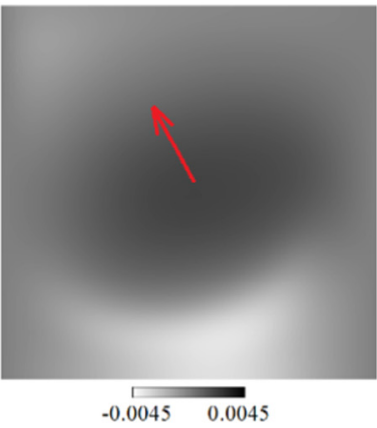

(b) $\tau=\tau_{0}+\tau_{\mathrm{p}} / 4$

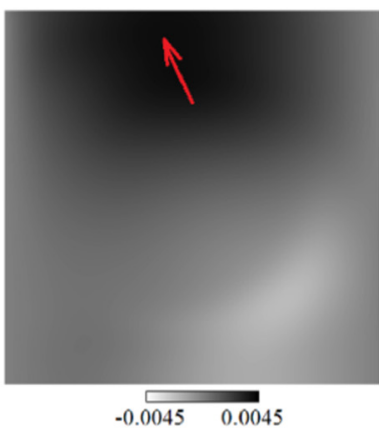

(c) $\tau=\tau_{0}+\tau_{\mathrm{p}} / 2$

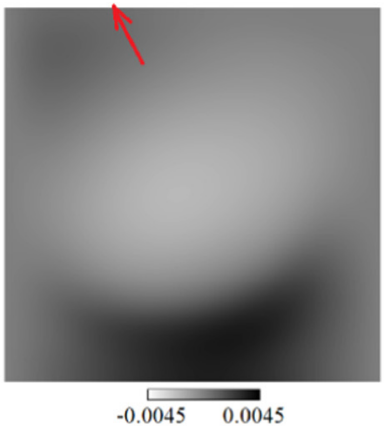

(d) $\tau=\tau_{0}+3 \tau_{\mathrm{p}} / 4$

Fig. 7. Snapshots of temperature fluctuation in the $R-Z$ plane of $\theta=0$ at $M a=500$ and $B i=0.5$ at every quarter-period of oscillation for $3 D$ oscillatory flow.
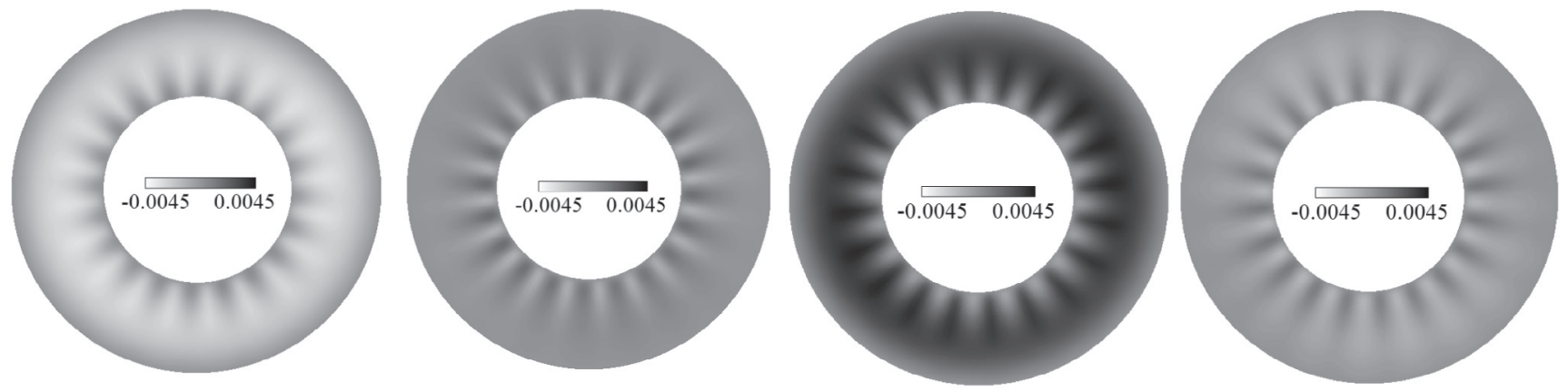

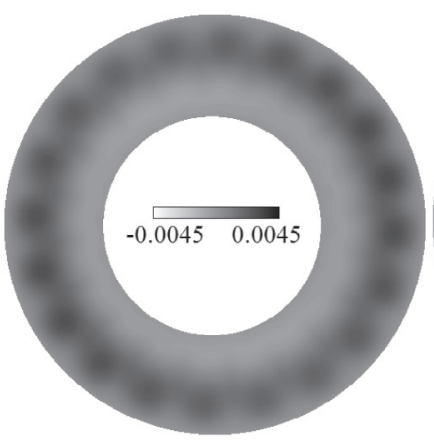

(a) $\tau=\tau_{0}$

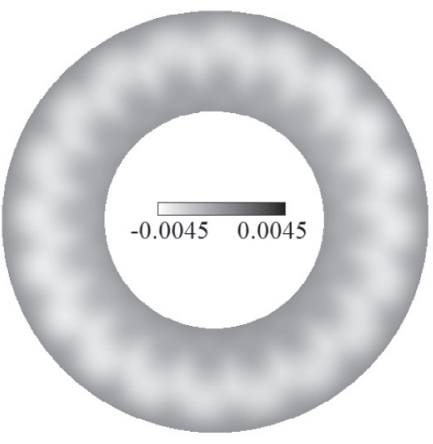

(b) $\tau=\tau_{0}+\tau_{\mathrm{p}} / 4$

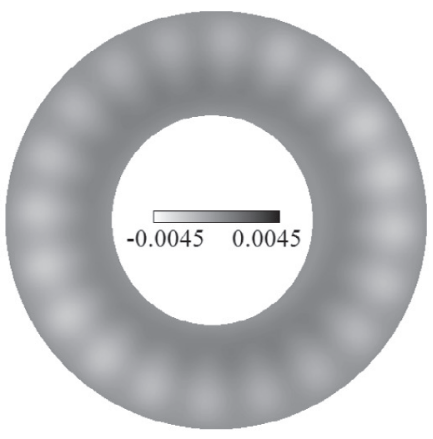

(c) $\tau=\tau_{0}+\tau_{\mathrm{p}} / 2$

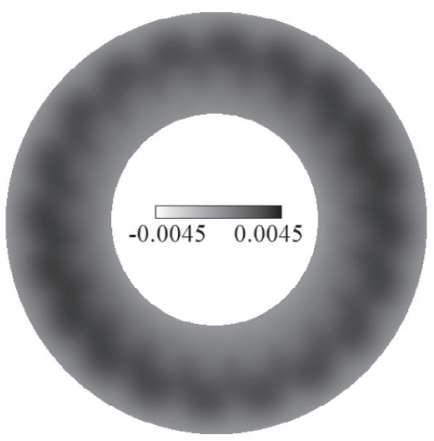

(d) $\tau=\tau_{0}+3 \tau_{\mathrm{p}} / 4$

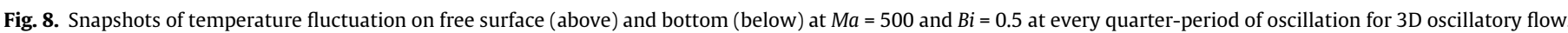


temperature fluctuation is dominant. Fig. 9 shows the evolution of free surface temperature with time at $R=1.17,1.5$ and 1.83 , respectively. Obviously, the azimuthal temperature fluctuation is dominant near the inner wall, and the azimuthal positions of the peaks and the troughs remain unchanged. Therefore, the spacetime diagram (STD) along a circumference of surface temperature consists of a set of vertically discontinuous lines. Near the outer wall, the radial temperature fluctuation is dominant and axisymmetric, so the STD consists of a set of horizontal straight lines. It is just the opposite at the bottom of the annular pool, that is, the radial temperature gradient is main near the inner wall, and the azimuthal temperature fluctuation is dominate near the outer wall.

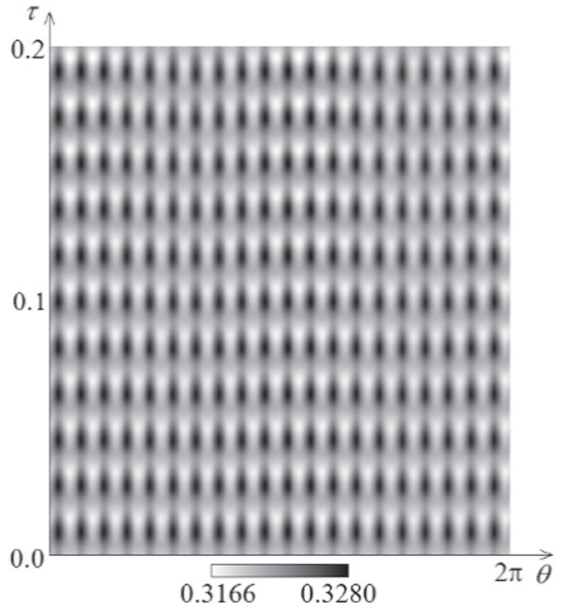

(a) $R=1.17$

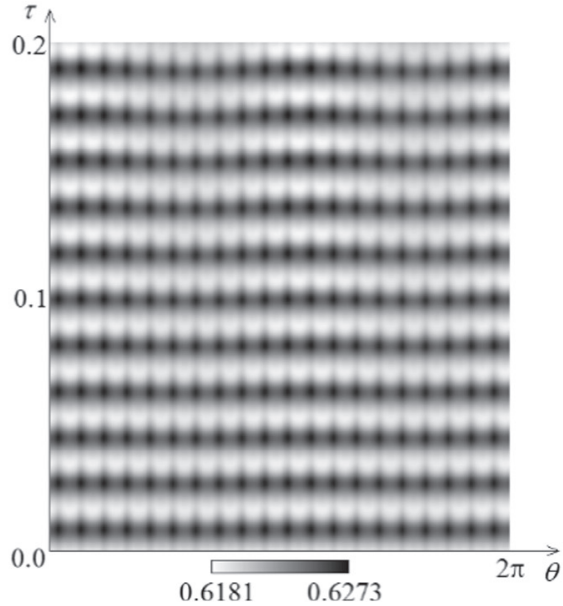

(b) $R=1.50$

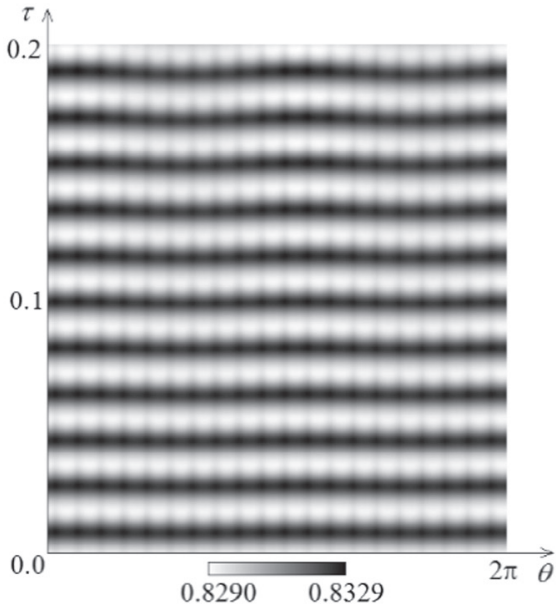

(c) $R=1.83$

Fig. 9. Variations of surface temperature distribution on free surface with time at $M a=500$ and $B i=0.5$ when $R=1.17,1.50$ and 1.83 , respectively.
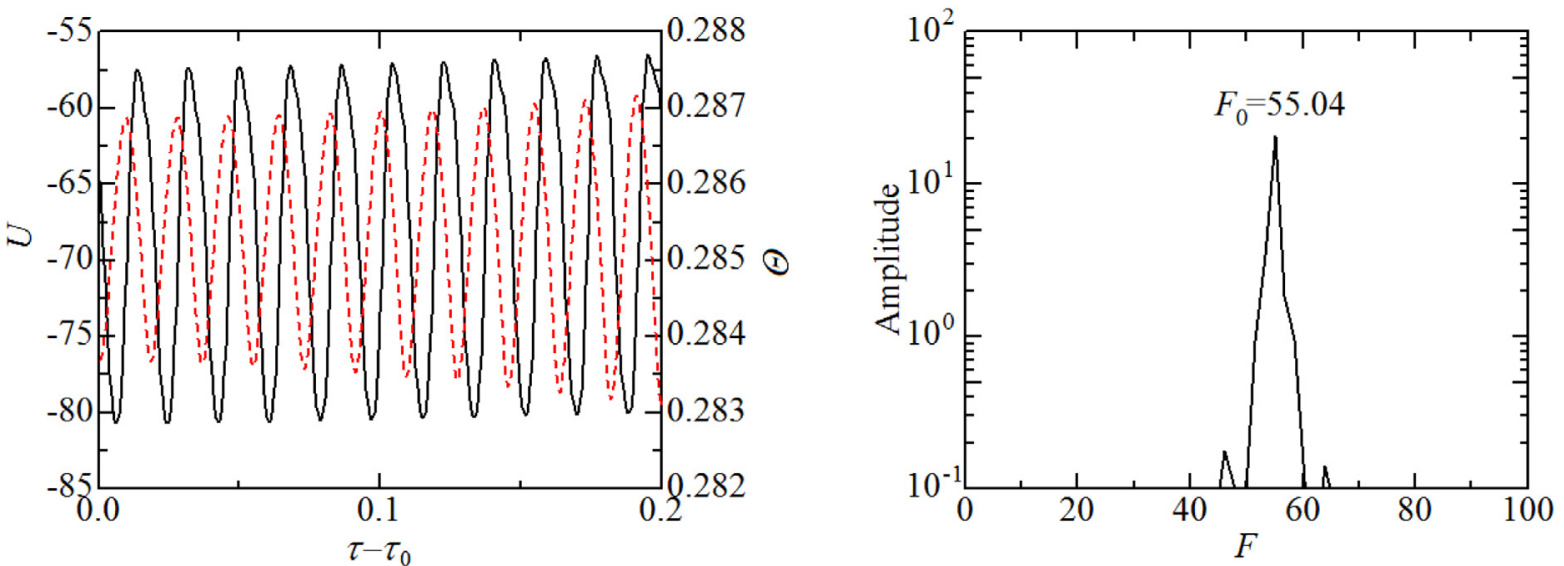

(a) $R=1.17$ and $Z=0.9$
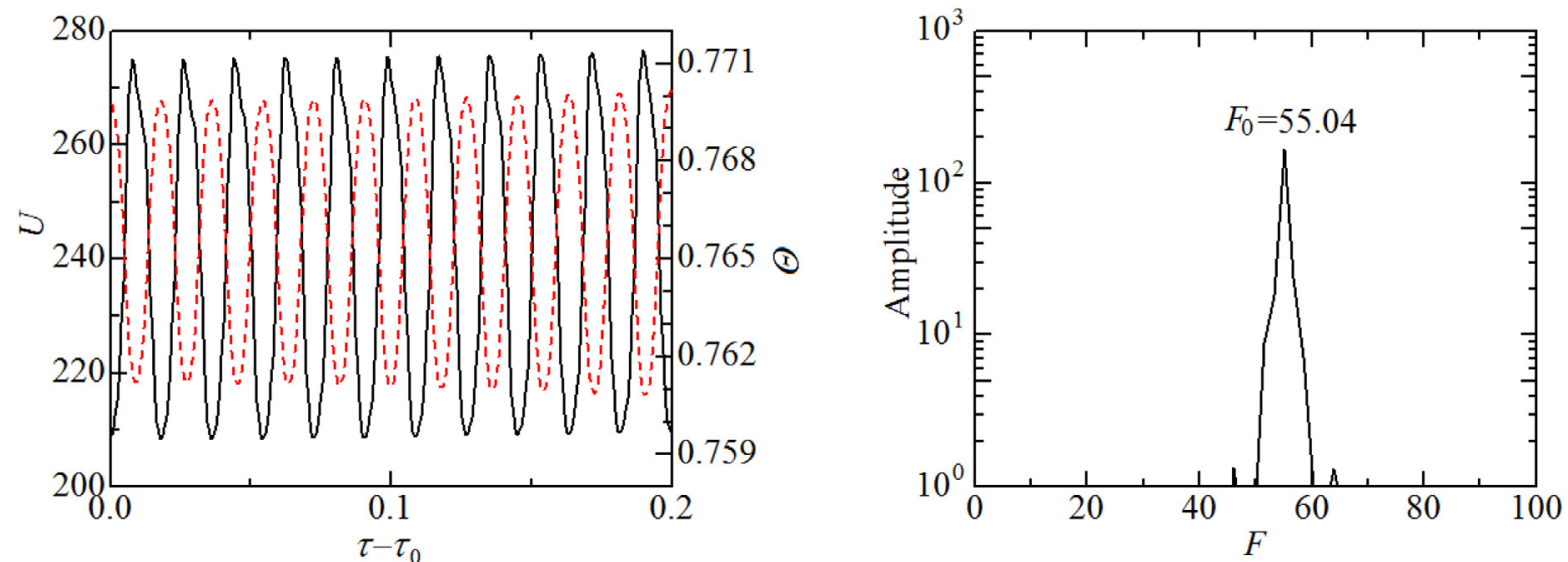

(b) $R=1.83$ and $Z=0.15$

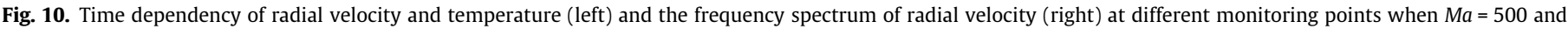
$B i=0.5$. Solid lines: radial velocity; dotted lines: temperature. 

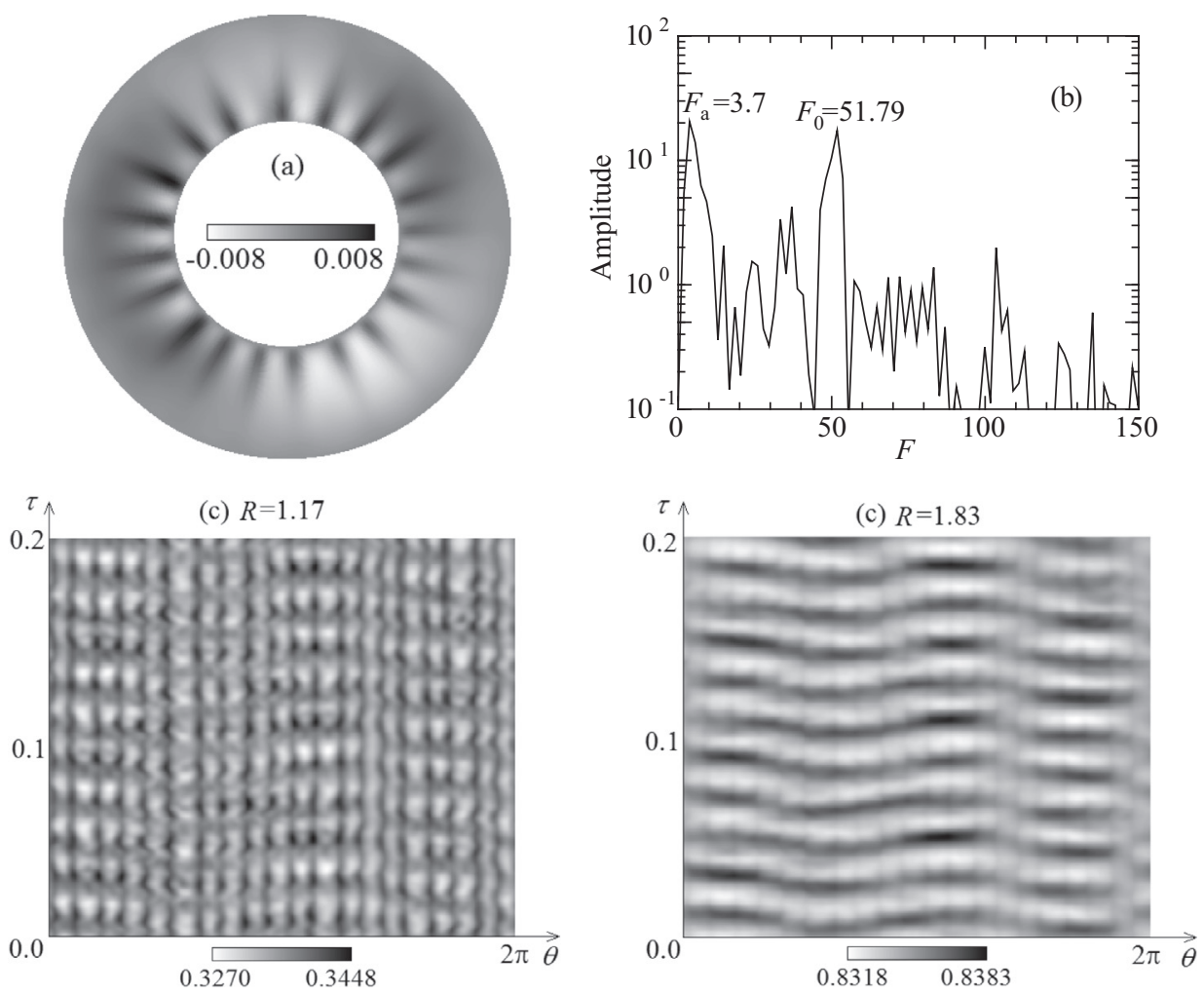

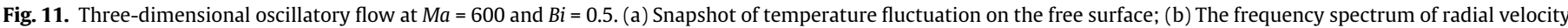
at $R=1.5$ on free surface; (c) Variations of surface temperature distribution with time on free surface at $R=1.17$ (left) and 1.83 (right).

Fig. 10 shows the time dependency of radial velocity and temperature and the frequency spectrum of radial velocity at different monitoring points when $M a=500$ and $B i=0.5$. Obviously, both velocity and temperature fluctuations have strong periodicity, but the fluctuation between them is not synchronous, and there is a certain phase difference. Near the free surface and the inner wall, the phase difference is $(3 / 7) \pi$, and near the bottom and the outer wall, the phase difference is $\pi$. According to the spectrum, although the phase difference between velocity fluctuation and temperature fluctuation varies in different positions of the annular liquid pool, the dimensionless fluctuation frequency is always the same, which is $F_{0}=55.04$. On the other hand, it can be found that the amplitudes of the temperature and velocity fluctuations near the bottom are larger than those near the free surface by comparison of Fig. 10 (a) and (b).

With the increase of Marangoni number, the flow on the $R-Z$ plane is enhanced, and the oscillation process becomes more complex. Fig. 11 gives the snapshot of temperature fluctuation, the frequency spectrum of radial velocity at a monitoring point and the evolution of surface temperature distribution with time at $R=1.17$ and 1.83 when $M a=600$ and $B i=0.5$. At this time, the temperature fluctuation along the azimuthal direction on the free surface is no longer uniform at any time, but the temperature at some azimuthal positions is higher, and it is lower at other positions. The azimuthal positions of the high and low temperature regions also change alternately, which leads to two dominant frequencies on the spectrogram, namely $F_{0}=51.79$ and $F_{\mathrm{a}}=3.70 . F_{0}$ is the oscillation frequency of the large cell on the $R-Z$ plane, while $F_{\mathrm{a}}$ reflects the alternation of the azimuthal positions of the high and low temperature regions. On the other hand, it can be seen from Fig. 11(c) that the azimuthal temperature fluctuation near the free surface will be no longer stable and there exists a small oscillation along the azimuthal direction. Therefore, the STD at $R=1.17$ is composed of a set of vertical twists and turns. At the same time, the axisym- metric radial temperature fluctuation near the outer wall is no longer symmetrical, so the horizontal lines on the STD at $R=1.83$ also begin to tilt and fracture. When Marangoni number increases further, thermocapillary convection will be bifurcated into chaotic flow.

\section{Conclusions}

A series of three-dimensional numerical simulations are performed to analyze clearly the flow pattern transition and the destabilization mechanism of thermocapillary convection for low Prandtl number fluid in a deep annular pool when surface heat dissipation is taken into account. From the obtained results, the following conclusions can be formulated:

(1) With the increase of Marangoni number, there are two types of flow pattern transition: the first is axisymmetric steady flow into 3D steady flow, and the second is 3D steady flow into 3D oscillation flow after the flow destabilization.

(2) The critical Marangoni number of flow pattern transition decreases slightly with the increase of Biot number. The flow destabilization is caused by the sudden change of flow direction near the upper part of inner wall and the lower part of outer wall. The maximum temperature and velocity fluctuations appear near the lower part of outer wall.

(3) With the increase of Biot number, the azimuthal temperature fluctuation on the free surface gradually shrinks to the inner wall, and the temperature fluctuation region decreases. However, the temperature fluctuation region at the bottom remains almost unchanged, but the fluctuation amplitude increases. Under the same Marangoni number, the variation of wave number will not be more than 2 when Biot number increases from 0 to 1 . 


\section{Conflict of interest}

The authors declare that there is no conflict of interest.

\section{Acknowledgement}

This work is supported by National Natural Science Foundation of China (Grant No. 51776022, 11532015).

\section{Appendix A. Supplementary material}

Supplementary data associated with this article can be found, in the online version, at https://doi.org/10.1016/j.ijheatmasstransfer. 2018.05.120.

\section{References}

[1] H.M. Li, W.Y. Shi, M.K. Ermakov, Thermocapillary flow instabilities of medium Prandtl number liquid in rotating annular pools, Int. J. Therm. Sci. 120 (2017) 233-243.

[2] C. Bach, D. Schwabe, Surface waves in thermocapillary flow - revisited, Eur. Phys. J. Special Topics 224 (2015) 319-340.

[3] A.A. Avramenko, I.V. Shevchuk, S. Harmand, A.I. Tyrinov, Thermocapillary instability in an evaporating two-dimensional thin layer film, Int. J. Heat Mass Transf. 91 (2015) 77-88.

[4] S.P. Aktershev, Stability of the heated liquid film in the presence of the thermocapillary effect, Thermophys. Aeromech. 20 (1) (2013) 1-16.

[5] B. Sobac, D. Brutin, Thermocapillary instabilities in an evaporating drop deposited onto a heated substrate, Phys. Fluids 24 (3) (2012) 032103.

[6] M.K. Smith, S.H. Davis, Instabilities of dynamic thermocapillary liquid layers. 1. Convective instabilities, J. Fluid Mech. 132 (1983) 119-144.

[7] M.K. Smith, S.H. Davis, Instabilities of dynamic thermocapillary liquid layers. 2. Surface-wave instabilities, J. Fluid Mech. 132 (1983) 145-162.

[8] M.K. Smith, Instability mechanisms in dynamic thermocapillary liquid layers, Phys. Fluids 29 (10) (1986) 3182-3186.

[9] A. Zebib, G.M. Homsy, E. Meiburg, High Marangoni number convection in a square cavity, Phys. Fluids 28 (1985) 3467-3476.

[10] H. Ben Hadid, B. Roux, Thermocapillary convection in long horizontal layers of low- Prandtl - number melts subject to a horizontal temperature gradient, J. Fluid Mech. 221 (1990) 77-103.

[11] J.F. Mercier, C. Normand, Buoyant-thermocapillary instabilities of differentially heated liquid layers, Phys. Fluids 8 (1996) 1443-1445.

[12] L. Peng, Y.R. Li, W.Y. Shi, N. Imaishi, Three-dimensional thermocapillarybuoyancy flow of silicone oil in a differentially heated annular pool, Int. J. Heat. Mass Transf. 50 (2007) 872-880.

[13] D. Villers, J.K. Platten, Coupled buoyancy and Marangoni convection in acetone: experiments and comparison with numerical simulations, J. Fluid Mech. 234 (1991) 487-510.

[14] P. Zhu, L. Duan, Q. Kang, Transition to chaos in thermocapillary convection, Int. J. Heat Mass Transf. 57 (2013) 457-464.

[15] P. Gillon, G.M. Homsy, Combined thermocapillary-buoyancy convection in a cavity: an experimental study, Phys. Fluids 8 (1996) 2953-2963.

[16] J.C. Chen, Y.R. Li, J.J. Yu, L. Zhang, C.M. Wu, Flow pattern transition of thermalsolutal capillary convection with the capillary ratio of -1 in a shallow annular pool, Int. J. Heat Mass Transf. 95 (2016) 1-6.
[17] J.J. Yu, Y.R. Li, C.M. Wu, J.C. Chen, Three-dimensional thermocapillarybuoyancy flow of a binary mixture with soret effect in a shallow annular pool, Int. J. Heat Mass Transf. 90 (2015) 1071-1081.

[18] J.J. Yu, D.F. Ruan, Y.R. Li, J.C. Chen, Experimental study on thermocapillary convection of binary mixture in a shallow annular pool with radial temperature gradient, Exp. Therm. Fluid Sci. 61 (2015) 79-86.

[19] J. Burguete, N. Mukolobwiez, F. Daviaud, N. Garnier, A. Chiffaudel, Buoyantthermocapillary instabilities in extended liquid layers subjected to a horizontal temperature gradient, Phys. Fluids 13 (2001) 2773-2787.

[20] S. Benz, D. Schwabe, The three-dimensional stationary instability in dynamic thermocapillary shallow cavities, Exp. Fluids 31 (2001) 409-416.

[21] F. Daviaud, J.M. Vince, Traveling waves in a fluid layer subjected to a horizontal temperature gradient, Phys. Rev. E 48 (1993) 4432-4436.

[22] N. Garnier, A. Chiffaudel, Two dimensional hydrothermal waves in an extend cylindrical vessel, Eur. Phys. J. B 19 (2001) 87-95.

[23] H.C. Kuhlmann, S. Albensoeder, Three-dimensional flow instabilities in a thermocapillary-driven cavity, Phys. Rev. E 77 (2008) 36303.

[24] L.J. Peltier, S. Biringen, Time-dependent thermocapillary convection in a rectangular cavity - numerical results for a moderate Prandtl number fluid, J. Fluid Mech. 257 (1993) 339-357.

[25] V. Sab, H. Kuhlmann, H. Rath, Investigation of three-dimensional thermocapillary convection in a cubic container by a multi-grid method, Int. J. Heat Mass Transf. 39 (1996) 603-613.

[26] C.J. Jing, N. Imaishi, S. Yasuhiro, Y. Miyazawa, Three-dimensional numerical simulation of spoke pattern in oxide melt, J. Cryst. Growth 200 (1990) 204212.

[27] B.C. Sim, A. Zebib, Effect of free surface heat loss and rotation on transition to oscillatory thermocapillary convection, Phys. Fluids 14 (2002) 225-231.

[28] Y. Kamotani, S. Ostrach, J. Masud, Microgravity experiments on oscillatory thermocapillary flow in cylindrical containers, J. Fluid Mech. 410 (2000) 211233.

[29] D. Schwabe, A. Zebib, B.C. Sim, Oscillatory thermocapillary convection in open cylindrical annuli. Part 1. Experiments under microgravity, J. Fluid Mech. 491 (2003) 239-258.

[30] B.C. Sim, A. Zebib, D. Schwabe, Oscillatory thermocapillary convection in open cylindrical annuli. Part 2. Simulations, J. Fluid Mech. 491 (2003) 259-274.

[31] H.F. Oztop, K. Lioua, B.M. Naceur, K. Al-Salem, Numerical study of threedimensional combined buoyancy and thermocapillary convection and evaluation of entropy generation, Int. J. Numer. Meth. Heat Fluid Flow 24 (2014) 148-168.

[32] R.E. Sakhy, K.E. Omari, Y. Le Guer, S. Blancher, Rayleigh-Bénard -Marangoni convection in an open cylindrical container heated by a non-uniform flux, Int. J. Therm. Sci. 86 (2014) 198-209.

[33] S. Hoyas, P. Fajardo, A. Gil, M.J. Perez-Quiles, Analysis of bifurcations in a Bénard-Marangoni problem: gravitational effects, Int. J. Heat Mass Transf. 73 (2014) 33-41.

[34] S. Hoyas, P. Fajardo, A. Gil, M.J. Perez-Quiles, Influence of geometrical parameters on the linear stability of a Bénard-Marangoni problem, Phys. Rev. E 93 (2016) 043105.

[35] A.J. Torregrosa, S. Hoyas, M.J. Perez-Quiles, J.M. Mompo-Laborda, Bifurcation diversity in an annular pool heated from below: Prandtl and Biot numbers effects, Commun. Comput. Phys. 13 (2013) 428-441.

[36] L. Zhang, Y.R. Li, C.M. Wu, Effect of surface heat dissipation on thermocapillary convection of low Prandtl number fluid in a shallow annular pool, Int. J. Heat Mass Transf. 110 (2017) 660-667.

[37] L. Zhang, Y.R. Li, C.M. Wu, Q.S. Liu, Flow bifurcation routes to chaos of thermocapillary convection for low Prandtl number fluid in shallow annular pool with surface heat dissipation, Int. J. Therm. Sci. 125 (2018) 23-33.

[38] Y.R. Li, L. Peng, Y. Akiyama, N. Imaishi, Three-dimensional numerical simulation of thermocapillary flow of moderate Prandtl number fluid in annular pool, J. Cryst. Growth 259 (2003) 374-387. 